\title{
Hydrodynamic gradient expansion in linear response theory
}

\author{
Michal P. Heller,$^{1,2, *}$ Alexandre Serantes $\odot,{ }^{2, \dagger}$ Michał Spaliński, ${ }^{2,3, \ddagger}$ Viktor Svensson $\odot,{ }^{2,1, \S}$ and Benjamin Withers ${ }^{4, \|}$ \\ ${ }^{1}$ Max Planck Institute for Gravitational Physics (Albert Einstein Institute), \\ 14476 Potsdam-Golm, Germany \\ ${ }^{2}$ National Centre for Nuclear Research, 02-093 Warsaw, Poland \\ ${ }^{3}$ Physics Department, University of Biatystok, 15-245 Biatystok, Poland \\ ${ }^{4}$ Mathematical Sciences and STAG Research Centre, University of Southampton, Highfield, \\ Southampton SO17 1BJ, United Kingdom
}

(Received 21 August 2020; revised 26 April 2021; accepted 16 July 2021; published 2 September 2021)

A foundational question in relativistic fluid mechanics concerns the properties of the hydrodynamic gradient expansion at large orders. We establish the precise conditions under which this gradient expansion diverges for a broad class of microscopic theories admitting a relativistic hydrodynamic limit, in the linear regime. Our result does not rely on highly symmetric fluid flows utilized by previous studies of heavy-ion collisions and cosmology. The hydrodynamic gradient expansion diverges whenever energy density or velocity fields have support in momentum space exceeding a critical momentum and converges otherwise. This critical momentum is an intrinsic property of the microscopic theory and is set by branch point singularities of hydrodynamic dispersion relations.

DOI: $10.1103 /$ PhysRevD.104.066002

\section{INTRODUCTION}

The goal of relativistic hydrodynamics is to provide an effective description of long-lived, long-wavelength excitations-hydrodynamic modes-which are generally expected to dominate nonequilibrium dynamics of collective states of quantum fields at macroscopic scales and sufficiently late times [1-4]. Understanding what exact scales and times these are has been a very active field of research of the past decade in connection with studies of collective phases of strong interactions in nuclear collisions at the Relativistic Heavy Ion Collider and the Large Hadron Collider, where relativistic hydrodynamics is the framework translating between the observed particle spectra and microscopic features such as characteristics of the initial state $[5,6]$. Related recent developments in relativistic hydrodynamics go well beyond the realm of nuclear physics and extend also to astrophysics [7-9], as well as to studies of strong gravity $[10,11]$.

*michal.p.heller@aei.mpg.de

†alexandre.serantesrubianes@ncbj.gov.pl

*michal.spalinski@ncbj.gov.pl

§viktor.svensson@aei.mpg.de

"b.s.withers@soton.ac.uk

Published by the American Physical Society under the terms of the Creative Commons Attribution 4.0 International license. Further distribution of this work must maintain attribution to the author(s) and the published article's title, journal citation, and DOI. Funded by SCOAP ${ }^{3}$.
Much progress occurred recently thanks to the effective field theory perspective formulated as a spacetime derivative expansion [12] as well as by using insights from linear response theory [13]. The effective field theory approach expresses expectation values of conserved currents in terms of derivatives of local classical fields such as the energy density and fluid velocity. The energy-momentum tensor is represented as a sum of all possible terms graded by the number of derivatives, starting with the perfect fluid contribution. By comparing this formal series to the analogous gradient expansion calculated in a microscopic theory, one can express the parameters appearing in the hydrodynamic series - transport coefficients-in terms of microscopic quantities. Interestingly, the gradient series evaluated on a solution of the evolution equations can have a vanishing radius of convergence at least in the case of highly symmetric flows describing rapidly expanding matter, as was discovered in holography [14-18], hydrodynamic models [19-21], and kinetic theory [22-25].

At the linearized level [26], the dynamics is governed by sums of harmonic contributions with complex frequencies which encode Fourier space singularities of retarded correlators. Imaginary parts of these frequencies capture effects of dissipation. Terms associated with frequencies which vanish at small momentum correspond to shear and sound hydrodynamic modes, while the rest represents transient phenomena [3]. Their dispersion relations are usually expressed as expansions in spatial momenta. Correspondingly, we choose the position space gradient expansion to be expressed in purely spatial derivatives, 
which can always be done. With this choice, the position space gradient expansion of the hydrodynamic constitutive relations is related to the shear and sound mode frequencies expanded in small spatial momentum. In Ref. [27] and later in Refs. [28,29], it was observed that the latter series have a finite nonzero radius of convergence, which reflects the presence of nonhydrodynamic modes. This parallels the fact that the Borel transform of the gradient expansion in an expanding plasma similarly reveals information about the nonhydrodynamic sectors.

The present article combines these two lines of research, which allows one to make for the first time generic statements about the convergence of the hydrodynamic gradient expansion across microscopic theories and models. In particular, we show that the convergence of the position space gradient expansion of the constitutive relations in the linearized regime is governed by the same mechanism that yields a finite radius of convergence of series expansions of hydrodynamic mode frequencies at small momentum: the radius of convergence of the dispersion relations is precisely the momentum scale which defines which flows possess a convergent asymptotic gradient expansion and which ones do not.

\section{HYDRODYNAMICS}

The expectation value of the energy-momentum tensor can be expressed as the perfect-fluid part plus corrections

$$
\left\langle T^{\mu \nu}\right\rangle=(\mathcal{E}+\mathcal{P}) U^{\mu} U^{\nu}+\mathcal{P} g^{\mu \nu}+\Pi^{\mu \nu} .
$$

In hydrodynamics, the correction $\Pi^{\mu \nu}$ is represented in terms of derivatives of the hydrodynamic fields which we take as the energy density $\mathcal{E}$ and flow velocity $U^{\mu}$ with $U \cdot U=-1$. The pressure $\mathcal{P}$ is related to $\mathcal{E}$ via an equation of state $[3,4]$.

We consider flat $d$-dimensional spacetime and use the Landau frame where $U_{\mu} \Pi^{\mu \nu}=0$. We focus on conformal and parity-invariant theories. Conformal symmetry forces $\Pi_{\mu}^{\mu}=0$ and $\mathcal{P}=\mathcal{E} /(d-1)$. The most general hydrodynamic $\Pi^{\mu \nu}$ takes now the form [30,31]

$$
\begin{aligned}
\Pi^{\mu \nu}= & -\eta \sigma^{\mu \nu}+\tau_{\pi} \eta \mathcal{D} \sigma^{\mu \nu} \\
& -\frac{1}{2} \theta_{1} \mathcal{D}_{\alpha} \mathcal{D}^{\alpha} \sigma^{\mu \nu}-\theta_{2} \mathcal{D}^{\langle\mu} \mathcal{D}^{\nu\rangle} \mathcal{D}_{\alpha} U^{\alpha}+\cdots,
\end{aligned}
$$

where the ellipsis denotes terms higher than third order and we display only terms surviving linearization. The angle brackets in (2) denote the tensors made symmetric, transverse, and traceless; $\mathcal{D}=U^{\mu} \partial_{\mu}$ and $\mathcal{D}^{\mu}=\left(g^{\mu \nu}+U^{\mu} U^{\nu}\right) \partial_{\nu}$ are, respectively, a comoving and a transverse derivative; $\sigma^{\mu \nu}=2 \mathcal{D}^{\langle\mu} U^{\nu\rangle}$ denotes the shear tensor; $\eta$ is the shear viscosity; $\tau_{\pi}$ is the relaxation time; and $\theta_{1}, \theta_{2}$ are thirdorder transport coefficients.
We focus on small perturbations away from thermal equilibrium; i.e., we consider

$$
U^{\mu}=(1, \mathbf{u})^{\mu} \quad \text { and } \quad \mathcal{E}=\mathcal{E}_{0}+\epsilon
$$

with $\left|\epsilon / \mathcal{E}_{0}\right|,\left|u_{l} u^{l}\right| \ll 1$. We denote spatial indices with latin letters and spatial vectors with bold font. It is useful to work in Fourier space with a plane-wave ansatz,

$$
\begin{aligned}
u^{i}(t, \mathbf{x}) & =\hat{u}^{i}(\mathbf{k}) e^{-i \omega t+i \mathbf{k} \cdot \mathbf{x}}, \\
\epsilon(t, \mathbf{x}) & =\hat{\epsilon}(\mathbf{k}) e^{-i \omega t+i \mathbf{k} \cdot \mathbf{x}} .
\end{aligned}
$$

The perturbations can be decomposed into shear and sound channel components [1], labeled here by $\perp$ and $\|$ subscripts. They are given by

$$
\hat{\mathbf{u}}_{\|}=\frac{\mathbf{k} \cdot \hat{\mathbf{u}}}{\mathbf{k}^{2}} \mathbf{k}, \quad \hat{\mathbf{u}}_{\perp}=\hat{\mathbf{u}}-\hat{\mathbf{u}}_{\|},
$$

with $\hat{\epsilon}=0$ vanishing in the shear channel. With no loss of generality, due to rotational invariance, we take

$$
\mathbf{k}=(0, \ldots, 0, k) \text {. }
$$

Conservation of the energy-momentum tensor together with the hydrodynamic constitutive relation (2) determines the frequencies $\omega$ appearing in (4) as functions of $k$. The dispersion relations take the form [30,31]

$$
\begin{aligned}
\tilde{\omega}_{\perp}= & -i \frac{\eta}{s T} k^{2}-i\left(\frac{\eta^{2} \tau_{\pi}}{s^{2} T^{2}}-\frac{\theta_{1}}{2 s T}\right) k^{4}+\cdots, \\
\tilde{\omega}_{\|}^{ \pm}= & \pm c_{s} k-i \Gamma k^{2} \mp \frac{\Gamma}{2 c_{s}}\left(\Gamma-2 c_{s}^{2} \tau_{\pi}\right) k^{3} \\
& -i\left(2 \Gamma^{2} \tau_{\pi}-\frac{(d-2)\left(\theta_{1}+\theta_{2}\right)}{2(d-1) s T}\right) k^{4}+\cdots,
\end{aligned}
$$

where the tilde means that these are frequencies in the hydrodynamic theory rather than in a microscopic theory. Here, $T$ and $s$ are the temperature and entropy density associated with $\mathcal{E}_{0}, c_{s}=1 / \sqrt{(d-1)}$ is the speed of sound, and $\Gamma=(d-2) /(d-1) \eta /(s T)$.

Calculations in holography [27-29] reveal that the series (7) have a finite and nonzero radius of convergence, with evidence going back to the studies of causal second-order hydrodynamics in Ref. [12]. In physically interesting cases, linear response theory shows that, apart from the hydrodynamic modes, there are additional excitations that are short lived, i.e., whose complex frequency $\omega(k)$ has a nonvanishing imaginary part even as $k \rightarrow 0[12,13,32,33]$. Explicit calculations in several representative cases show that the radius of convergence of hydrodynamic dispersion relations is set by the magnitude $k_{*}$ of a (possibly complex) momentum for which the frequency of a hydrodynamic 
mode coincides with that of a nonhydrodynamic one at a branch point of $\omega(k)$ [27-29].

\section{CONSTITUTIVE RELATIONS}

To exploit the known properties of $\omega(k)$, we parametrize the gradient expansion of $\Pi^{\mu \nu}$ using only spatial derivatives. A different choice is explored in Appendix B.

The most general form of $\Pi^{\mu \nu}$ can be constructed from three elementary tensorial structures that are, respectively,

$$
\begin{gathered}
\sigma_{j l}=\left(\partial_{j} u_{l}+\partial_{l} u_{j}-\frac{2}{d-1} \delta_{j l} \partial_{r} u^{r}\right), \\
\pi_{j l}^{\epsilon}=\left(\partial_{j} \partial_{l}-\frac{1}{d-1} \delta_{j l} \partial^{2}\right) \epsilon, \\
\pi_{j l}^{u}=\left(\partial_{j} \partial_{l}-\frac{1}{d-1} \delta_{j l} \partial^{2}\right) \partial_{r} u^{r} .
\end{gathered}
$$

The last of these appears already in Refs. [34,35] (see also Ref. [29]). ${ }^{1}$ With no loss of generality, we write the constitutive relations as

$$
\Pi_{j l}=-A\left(\partial^{2}\right) \sigma_{j l}-B\left(\partial^{2}\right) \pi_{j l}^{u}-C\left(\partial^{2}\right) \pi_{j l}^{\epsilon}
$$

and $\Pi_{t t}=\Pi_{t i}=0 . A, B$, and $C$ are infinite series in spatial Laplacians,

$$
A=\sum_{n=0}^{\infty} a_{n}\left(-\partial^{2}\right)^{n},
$$

and the $a_{n}$ are transport coefficients, with similar expressions for $B$ and $C$ involving transport coefficients $b_{n}$ and $c_{n}$. In principle, $A, B$, and $C$ could also depend on $\partial_{t}$, but in the hydrodynamic gradient expansion, one can use the conservation equations to replace temporal derivatives by spatial ones in a systematic way. ${ }^{2}$

It follows from (9) that each even order in gradients introduces one new transport coefficient, while each odd order higher than 1 introduces two. We find it remarkable that such a simple argument implies that the number of independent transport coefficients at a given order in the gradient expansion of linearized hydrodynamics does not grow with the order but is limited.

An analogous situation occurs in the series expansions of $\omega_{\perp}, \omega_{\|}^{ \pm}$around $k=0$. Since $\omega_{\|}^{+}, \omega_{\|}^{-}$obey the relation $\omega_{\|}^{+}(k)=-\omega_{\|}^{-}(k)^{*}$, their series coefficients are not

\footnotetext{
${ }^{1}$ References [34,35] are part of a program studying the stress tensor of holographic theories in the linear response regime (see also Refs. [36-38]). In contrast, we study hydrodynamic constitutive relations perturbatively in spatial gradients, and without restriction to a particular microscopic model.

${ }^{2}$ See Appendix A of Ref. [30].
}

independent. These coefficients are real for odd powers of $k$ and purely imaginary for even powers of $k . \omega_{\perp}$ is given by a series expansion in $k^{2}$ with purely imaginary coefficients. Therefore, each even order in (7) introduces two new real parameters, while each odd order introduces just one. This counting matches the number of independent transport coefficients in (9) and suggests that it is possible to express $a_{n}, b_{n}$, and $c_{n}$, see (10), in terms of the hydrodynamic dispersion relations (7).

\section{MATCHING}

We now show explicitly that there is a direct relation between $A, B$, and $C$ defined in (9) and the hydrodynamic dispersion relations (7).

For the shear mode, with the wave vector choice we made in (6), the only nonzero components of $\sigma_{j l}$ are

$$
\sigma_{1, d-1}=\sigma_{d-1,1}=i k u_{1}
$$

where we have taken $\mathbf{u}=\left(u_{1}, 0, \ldots, 0\right)$ with no loss of generality due to rotational invariance. $\pi_{j l}^{u}$ and $\pi_{j l}^{\epsilon}$ vanish identically for this mode since $\partial_{i} u^{i}=\epsilon=0$.

The conservation of the energy-momentum tensor (1), in combination with the hydrodynamic constitutive relation (9), predicts the following dispersion relation:

$$
\tilde{\omega}_{\perp}(k)=-i \frac{1}{s T} \sum_{n=0}^{\infty} a_{n} k^{2 n+2} .
$$

Demanding that $\tilde{\omega}_{\perp}(k)$ agrees with the microscopic shear hydrodynamic mode $\omega_{\perp}$ at every order in an expansion around $k^{2}=0$ fixes the $a_{n}$ coefficients to be

$$
a_{n}=\left[k^{2 n+2}\right]\left(i s T \omega_{\perp}\right),
$$

where the notation $\left[k^{p}\right](f)$ denotes the coefficient of $k^{p}$ in the series expansion of $f$ around $k=0$.

With $A\left(\partial^{2}\right)$ fixed, we determine $B\left(\partial^{2}\right)$ and $C\left(\partial^{2}\right)$ by considering the sound mode. Now, $\mathbf{u}=\left(0, \ldots, 0, u_{d-1}\right)$, $\epsilon \neq 0$, and

$$
\pi_{j l}^{u}=-\frac{1}{2} k^{2} \sigma_{j l}
$$

Furthermore, the only nonzero components of $\sigma_{j l}$ and $\pi_{j l}^{\epsilon}$ are

$$
\begin{gathered}
\sigma_{j j}=-\frac{2}{d-1} i k u_{d-1}, \quad j=1 \ldots d-2, \\
\sigma_{d-1, d-1}=\frac{2(d-2)}{d-1} i k u_{d-1} \\
\pi_{j j}^{\epsilon}=\frac{1}{d-1} k^{2} \epsilon, \quad j=1 \ldots d-2,
\end{gathered}
$$




$$
\pi_{d-1, d-1}^{\epsilon}=-\frac{d-2}{d-1} k^{2} \epsilon
$$

In the end, the conservation equations reduce to

$$
\begin{gathered}
-i \omega \epsilon+i k s T u_{d-1}=0, \\
-i \omega s T u_{d-1}+\frac{1}{d-1} i k \epsilon \\
+\frac{d-2}{d-1} \sum_{n=0}^{\infty}\left(2 a_{n}-b_{n-1}\right) k^{2 n+2} u_{d-1} \\
+\frac{d-2}{d-1} \sum_{n=0}^{\infty} i c_{n} k^{2 n+3} \epsilon=0,
\end{gathered}
$$

where we have introduced $b_{-1} \equiv 0$ for brevity. Note that the conservation equation (16a) does not depend on transport coefficients as a result of our frame choice. Equation (16) has two solutions, $\tilde{\omega}_{\|}^{+}(k)$ and $\tilde{\omega}_{\|}^{-}(k)$, given as series expansions around $k=0$, whose coefficients depend on $a_{n}, b_{n}$, and $c_{n}$. Demanding that these quantities agree with the microscopic sound modes $\omega_{\|}^{+}(k)$ and $\omega_{\|}^{-}(k)$, the matching conditions for $b_{n}$ and $c_{n}$ are

$$
\begin{array}{r}
b_{n}=\left[k^{2 n+4}\right]\left(-i \frac{d-1}{d-2} s T\left(\omega_{\|}^{+}+\omega_{\|}^{-}\right)+2 i s T \omega_{\perp}\right), \\
c_{n}=\left[k^{2 n+4}\right]\left(-\frac{k^{2}}{d-2}-\frac{d-1}{d-2} \omega_{\|}^{+} \omega_{\|}^{-}\right) .
\end{array}
$$

The coefficients $a_{n}, b_{n}$, and $c_{n}$ are directly related to the transport coefficients defined in the standard way. Up to third order in gradients, one has

$a_{0}=\eta, \quad a_{1}=\frac{\eta^{2} \tau_{\pi}}{s T}-\frac{1}{2} \theta_{1}, \quad c_{0}=\frac{2 \eta \tau_{\pi}}{(d-1) s T}$,

$b_{0}=\theta_{2}-\frac{2(d-3) \eta^{2} \tau_{\pi}}{(d-1) s T}$.

The explicit relation between hydrodynamic dispersion relations (7) and hydrodynamic constitutive relations as encapsulated by (13) and (17) will directly lead to our main result on the convergence of the gradient expansion in linearized relativistic hydrodynamics. Its importance stems from the fact that it connects well-studied hydrodynamic dispersion relations as series in small $k$ with position-space hydrodynamic constitutive relations.

\section{LARGE-ORDER BEHAVIOR}

The analytic properties of the dispersion relations can be used to constrain the growth of transport coefficients. We expect that in a microscopic theory which respects relativistic causality, the hydrodynamic dispersion relations $\omega_{\perp}(k)$ and $\omega_{\|}^{ \pm}(k)$ have at least one branch-point singularity in the complex $k$-plane. This is realized in theories of causal hydrodynamics and holography, and in Appendix A, we provide an additional argument in favor of it. It implies that $\omega_{\perp}(k)$ and $\omega_{\|}(k)$ cannot be polynomials in $k$, so the hydrodynamic gradient expansion (9) following from the matching conditions (13) and (17) must contain an infinite number of terms. Moreover, the transport coefficients $a_{n}, b_{n}$, and $c_{n}$ grow geometrically in a manner controlled by the position of the branch points closest to $k=0$ [39],

$$
\lim _{n \rightarrow \infty}\left|a_{n}\right|^{\frac{1}{n}}=\left|k_{*}^{(A)}\right|^{-2},
$$

where $\left|k_{*}^{(A)}\right|$ denotes the modulus of the branch point location, and analogous expressions hold for $b_{n}$ and $c_{n}$. Note that $\left|k_{*}^{(A)}\right|,\left|k_{*}^{(B)}\right|,\left|k_{*}^{(C)}\right|$ correspond to the closest branch point between $\omega_{\perp}$ and $\omega_{\|}$as dictated by (13) and (17). The power appearing on the right-hand side of (19) is due to the fact that the transport coefficients are coefficients of a Taylor series in $k^{2}$.

\section{CONVERGENCE}

The convergence properties of the series (9) depend on the behavior of the transport coefficients $a_{n}, b_{n}$, and $c_{n}$ as well as on the particular solution $\epsilon$ and $\mathbf{u}$. Here, we show that the support in momentum space of the latter plays a crucial role in determining the radius of convergence of the gradient expansion. We focus on square-integrable functions, thus excluding trivial cases for which the gradient expansion truncates.

We assume that the flow is homogeneous in the $x^{1}, \ldots, x^{d-2}$ directions and define $x \equiv x^{d-1}$. Furthermore, we take the Fourier transforms of $\epsilon(t, x)$ and $u^{i}(t, x), \hat{\epsilon}(t, k)$ and $\hat{u}^{i}(t, k)$, to vanish for $|k|>k_{\max }$. In the linearized regime, the support is time independent, and this condition is a restriction on the initial data.

According to the Paley-Wiener theorem [40], the Fourier transform of a square-integrable function $\hat{f}(k)$ supported in $|k| \leq k_{\max }$ is an entire function of exponential type $k_{\max }{ }^{3}$ For a function of this kind, it follows that [41]

$$
\limsup _{n \rightarrow \infty}\left|f^{(n)}(x)\right|^{\frac{1}{n}}=k_{\max } \cdot
$$

Consider now the $A$-contribution to (9). For a compactly supported $\hat{u}^{i}, \sigma_{j l}(t, x)$ will be of exponential type $k_{\max }$ for all times. Hence,

$$
\limsup _{n \rightarrow \infty}\left|\partial_{x}^{2 n} \sigma_{j l}(t, x)\right|^{\frac{1}{n}}=k_{\max }^{2} .
$$

\footnotetext{
${ }^{3}$ See Appendix A for the relevant mathematical background.
} 
Applying the root test results in the following convergence criterion for the $A$-contribution to (9):

$$
\limsup _{n \rightarrow \infty}\left|a_{n} \partial_{x}^{2 n} \sigma_{j l}(t, x)\right|^{\frac{1}{n}}=\frac{k_{\max }^{2}}{\left|k_{*}^{(A)}\right|^{2}}<1 .
$$

Analogous arguments apply to the remaining pieces of (9). Let us define

$$
k_{*}=\min \left\{\left|k_{*}^{(A)}\right|,\left|k_{*}^{(B)}\right|,\left|k_{*}^{(C)}\right| .\right.
$$

We are now ready to state our main result for a generic excitation: the gradient expansion is a convergent series if the support of the hydrodynamic perturbations and their time-derivatives is smaller than the microscopic momentum scale $k_{*}$. If the support exceeds $k_{*}$, then the series is divergent; this includes the case of data which is not compactly supported.

It follows that if the hydrodynamic series is convergent, then its data are compactly supported with support that does not exceed $k_{*}{ }^{4}$

Even if divergent, the partial sums of the gradient expansion only grow geometrically as long as the support of the hydrodynamic fields in $k$-space does not extend to infinity. If it does, this geometric divergence is enhanced to the factorial one known from the studies of expanding geometries [14,19-21,23,24,42].

For a flow without any symmetry restrictions, we can argue heuristically that the same convergence conditions hold. Let us focus again on the $A$-contribution to (9). Truncating the series to $N$ th-order results in

$$
-\int_{\mathbb{R}^{d-1}} d^{d-1} \mathbf{k}\left[\sum_{n=0}^{N} a_{n}\left(\mathbf{k}^{2}\right)^{n}\right] \hat{\sigma}_{i j}(t, \mathbf{k}) e^{i \mathbf{k} \cdot \mathbf{x}},
$$

where we have interchanged the order of summation and integration. According to (19), the partial sums appearing in (24) are convergent as $N \rightarrow \infty$, provided that they are evaluated at $|\mathbf{k}|<\left|k_{*}^{(A)}\right|$. Outside this $(d-1)$-dimensional sphere, we get a nonconvergent series. Hence, it seems natural to assume that the condition for (24) to converge as $N \rightarrow \infty$ is that the hydrodynamic variable $\hat{\mathbf{u}}$ does not have support past $\left|k_{*}^{(A)}\right|$. Analogous arguments would hold also for the $B$ - and $C$-pieces, supporting the fact that the convergence criterion spelled out before is fully general.

\section{ILLUSTRATIVE EXAMPLE}

For illustration, we consider a shear channel perturbation in Müller-Israel-Stewart theory [43-45],

\footnotetext{
${ }^{4}$ In the special case where only the shear channel is excited, the appropriate notion of $k_{*}=\left|k_{*}^{(A)}\right|$.
}

$$
\epsilon=0, \quad \mathbf{u}=\left(u_{1}(t, x), 0, \ldots, 0\right) .
$$

We emphasise that our results apply more generally, but this is a particularly simple model of equilibration compatible with relativistic causality featuring a hydrodynamic regime.

In this case, the only tensor structure contributing to (9) is the shear tensor, and the only nontrivial independent component of the constitutive relations is

$$
\Pi_{1, d-1}(t, x)=-\sum_{n=0}^{\infty} a_{n}(-1)^{n} \partial_{x}^{2 n+1} u_{1}(t, x) .
$$

The $a_{n}$ transport coefficients can be computed in closed form, since the shear hydrodynamic mode is known exactly [12],

$$
\omega_{\perp}(k)=i \frac{-1+\sqrt{1-4 D \tau_{\pi} k^{2}}}{2 \tau_{\pi}},
$$

where $D \equiv \eta /(s T)=(d-1) /(d-2) \Gamma$ is the diffusion constant. Müller-Israel-Stewart theory contains also a single nonhydrodynamic shear mode which differs from (27) by the sign of the square root. The final result for the $a_{n}$ coefficients is

$$
a_{n}=s T \mathcal{C}_{n} D^{n+1} \tau_{\pi}^{n}
$$

where $\mathcal{C}_{n}$ are the Catalan numbers. Therefore,

$$
\left|k_{*}^{(A)}\right|=\left(\lim \sup _{n \rightarrow \infty}\left|a_{n}\right|^{\frac{1}{n}}\right)^{-1 / 2}=1 / \sqrt{4 D \tau_{\pi}},
$$

which is also the location of the branch points of (27), where the hydrodynamic and the nonhydrodynamic modes collide.

The initial state of the system is fully specified by $u_{1}(0, x)$ and $\partial_{t} u_{1}(0, x)$. We take $u_{1}(0, x)=0$ and

$$
\partial_{t} \hat{u}_{1}(0, k)=\frac{1}{2 \pi} e^{-\frac{1}{2} \gamma^{2} k^{2}} \Theta\left(k_{\max }^{2}-k^{2}\right),
$$

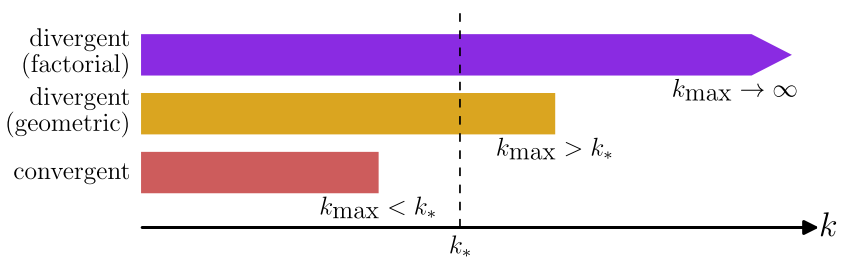

FIG. 1. The key quantity determining whether the gradient expansion diverges is the support of hydrodynamic fields in momentum space, $k_{\max }$, represented in this figure by the intervals. If the support extends to infinity, the expansion diverges factorially. If it exceeds $k_{*}$ but truncates at some finite momentum $k_{\max }$, it diverges geometrically. If $k_{\max }<k_{*}$, it converges. See Fig. 2 for an explicit example illustrating this general behavior. 

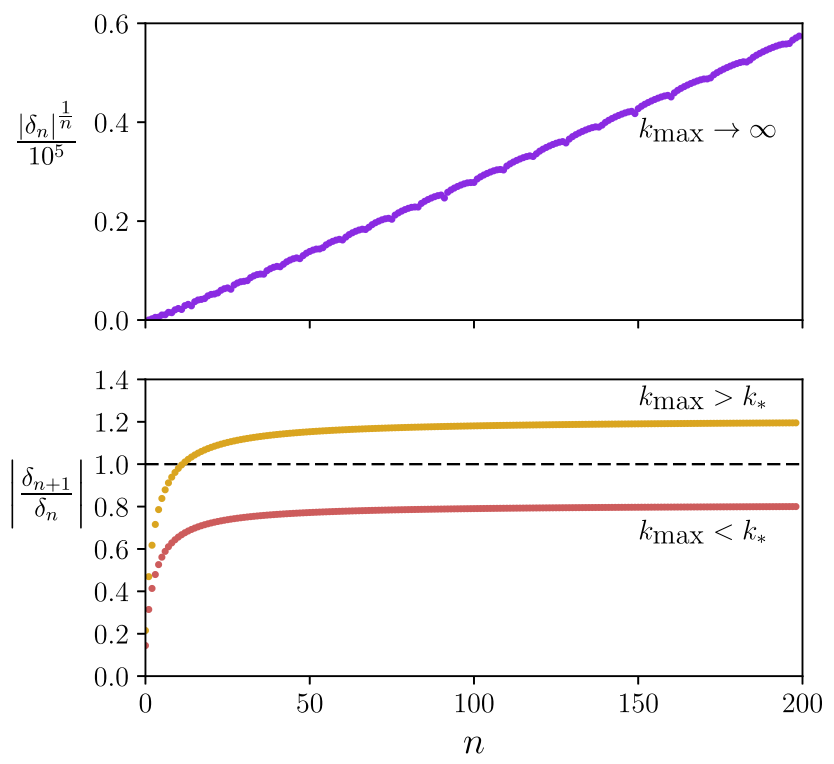

FIG. 2. Convergence tests applied to the gradient expansion (26), where $\delta_{n}$ denotes the $n$th contribution. We use $\gamma=0.1$ and consider $t=1, \quad x=0.5 \quad$ with $\quad s=T=\eta=\tau_{\pi}=1$ $\left(k_{*}=\left|k_{*}^{(A)}\right|=0.5\right.$; see footnote 4 ). Data point colors and topbottom ordering correspond to the cases described in Fig. 1. Upper figure: root test applied to $\delta_{n}$ when $k_{\max } \rightarrow \infty$. The geometric divergence of the gradient expansion is enhanced to a factorial one. Lower figure: ratio test applied to the solution with $k_{\max }=0.55$ (top) and 0.45 (bottom). The gradient expansion is convergent for $k_{\max }<k_{*}$ and geometrically divergent for $k_{\max }>k_{*}$.

where $\Theta$ is the Heaviside step function. As seen in Fig. 2, the position space gradient expansion is convergent for $k_{\max }^{2}<1 /\left(4 D \tau_{\pi}\right)$, geometrically divergent for $1 /\left(4 D \tau_{\pi}\right) \leq$ $k_{\max }^{2}<\infty$, and factorially divergent for $k_{\max } \rightarrow \infty$. This is exactly what is expected on the basis of our general analysis. For more details, see Ref. [46].

\section{CONCLUSIONS}

Our work reveals that hydrodynamics itself is neither convergent nor divergent; instead, such statements are conditional on the particular solution under consideration. We have provided a rigorous derivation of a general feature of hydrodynamics which one may phrase heuristically as "hydrodynamics breaks down when gradients become large." Furthermore, we quantified where the hydrodynamic series fails to converge for a general class of models.

Our detailed calculations reveal that the physics governing the convergence of position-space constitutive relations and the convergence of momentum-space dispersion relations are one and the same. In this way, we provide a unified perspective on two seemingly disparate lines of research represented by Refs. [14-25] and [27-29].

There are several important lessons that can be drawn from our work in relation to Bjorken flow. First, since we did not impose any particular symmetry, we have shown that the position space hydrodynamic series can still diverge even in the absence of the highly constraining symmetries of boost invariance. Second, while it is conceivable that nonlinear theories diverge due to a factorially growing number of transport coefficients at each order, here we show that this is not a necessary condition since we have at most two transport coefficients at each order and find divergence. Within an analogy with perturbative expansions in quantum mechanical systems dating back to Ref. [14], this is similar to renormalon rather than earlier anticipated instanton-related effects [47]. The continuation of these results in the presence of nonlinearities will be discussed in upcoming work [48].

The issue of the convergence of the hydrodynamic gradient expansion is often conflated with the issue of applicability of hydrodynamics for modeling microscopic theories. We have shown that the former is determined by support in momentum space; however, one can imagine a situation where nonhydrodynamic modes make a significant contribution in a microscopic theory even for states with support only at low momentum. Such a significant contribution would render hydrodynamics inapplicable even if convergent. On the other hand, even if a series diverges, it can provide a good description when optimally truncated. These observations suggest that there is no connection between the two issues at the linear level. It should be noted that this work enables a comprehensive study of optimal truncation-as a function of initial conditions-which we leave to future work.

It is very important that complete information about the nonhydrodynamic sector is encoded in the gradient series itself. In the case of an expanding plasma, this is very beautifully expressed by the phenomenon of resurgence [49], which makes it possible to extract the form of the full solution from the asymptotic series $[18,19,21]$. An analogous encoding of nonhydrodynamic data in the hydrodynamic sector is seen in the analytic continuation of dispersion relations [27]. Generalizations of these ideas based on developments reported in this article are the subject of Refs. [46,48].

\section{ACKNOWLEDGMENTS}

We would like to thank G. Dunne as well as participants of the workshop Foundational Aspects of Relativistic Hydrodynamics at Banff International Research Station, where this work was presented for the first time, for helpful discussions. We also have the pleasure to acknowledge comments on the draft from M. Baggioli, A. Buchel, A. Jansen, M. Lublinsky, J. Noronha, and C. Pantelidou. The Gravity, Quantum Fields and Information group at A. E. I. is supported by the Alexander von Humboldt Foundation and the Federal Ministry for Education and Research through the Sofja Kovalevskaja Award. A. S. and M. S. are supported by the Polish National Science Centre Grant No. 2018/29/B/ 
ST2/02457. B. W. is supported by a Royal Society University Research Fellowship.

\section{APPENDIX A: THE DISPERSION RELATIONS AND RELATIVISTIC CAUSALITY}

Our main objective in this Appendix is to provide additional arguments in favor of the two hypothesis regarding the behavior of the hydrodynamic dispersion relations put forward in the main text:

(1) $\omega(k)$ has at least one singularity in the complex $k$-plane.

(2) This singularity is a branch point.

We start by recalling that, under a metric fluctuation $\eta^{\mu \nu} \rightarrow \eta^{\mu \nu}+h^{\mu \nu}$, the response of the energy-momentum tensor expectation value in the thermal state is controlled by the retarded two-point function

$$
G^{\mu \nu, \alpha \beta}(t, \mathbf{x})=-i \Theta(t)\left\langle\left[T^{\mu, \nu}(t, \mathbf{x}), T^{\alpha \beta}(0,0)\right]\right\rangle
$$

as

$$
\begin{aligned}
& \delta\left\langle T^{\mu \nu}(t, \mathbf{x})\right\rangle \\
& \quad=-\frac{1}{2} \int_{\mathbb{R}^{1, d-1}} d t^{\prime} d^{d} \mathbf{x}^{\prime} G^{\mu \nu, \alpha \beta}\left(t-t^{\prime}, \mathbf{x}-\mathbf{x}^{\prime}\right) h_{\alpha \beta}\left(t^{\prime}, \mathbf{x}^{\prime}\right) .
\end{aligned}
$$

The expectation values are taken in the background thermal state. Defining

$$
G^{\mu \nu, \alpha \beta}(t, \mathbf{x})=\int_{\mathbb{R}^{1, d-1}} d \omega d^{d-1} \mathbf{k} e^{-i \omega t+i \mathbf{k} \cdot \mathbf{x}} \hat{G}^{\mu \nu, \alpha \beta}(\omega, \mathbf{k}),
$$

and similarly for $h^{\mu \nu}$, Eq. (A2) can be written as

$$
\begin{aligned}
& 2(2 \pi)^{-d} \delta\left\langle T^{\mu \nu}(t, \mathbf{x})\right\rangle \\
& =-\int_{\mathbb{R}^{1, d-1}} d \omega d^{d-1} \mathbf{k} e^{-i \omega t+i \mathbf{k} \cdot \mathbf{x}} \hat{G}^{\mu \nu, \alpha \beta}(\omega, \mathbf{k}) \hat{h}_{\alpha \beta}(\omega, \mathbf{k}) .
\end{aligned}
$$

Hydrodynamic and nonhydrodynamic frequencies appear as poles of $\hat{G}^{\mu \nu, \alpha \beta}(\omega, \mathbf{k})$, which, due to rotational invariance, only depend on $\mathbf{k}^{2}$. See Ref. [13] for a detailed discussion of how rotational invariance constrains the form of the retarded correlator. To discuss the interplay between relativistic causality and the analyticity properties of these frequencies, we consider the following setup: we imagine that our metric fluctuation is only active at $t=0$, and furthermore, we also assume that it only depends on $x^{d-1} \equiv x$,

$$
h^{\mu \nu}(t, \mathbf{x})=\delta(t) f^{\mu \nu}(x)
$$

In momentum space,

$$
\hat{h}^{\mu \nu}(\omega, \mathbf{k})=\frac{1}{2 \pi} \delta\left(k_{1}\right) \ldots \delta\left(k_{d-2}\right) \hat{f}^{\mu \nu}(k),
$$

where we have also defined $k \equiv k_{d-1}$. Hence,

$$
\begin{aligned}
& 2(2 \pi)^{1-d} \delta\left\langle T^{\mu \nu}(t, x)\right\rangle \\
& \quad=-\int_{\mathbb{R}^{1,1}} d \omega d k e^{-i \omega t+i k x} \hat{G}^{\mu \nu, \alpha \beta}(\omega, 0, \ldots, 0, k) \hat{f}_{\alpha \beta}(k) .
\end{aligned}
$$

Performing the integral with respect to $\omega$, we obtain

$$
\begin{aligned}
\delta\left\langle\hat{T}^{\mu \nu}(t, k)\right\rangle= & \sum_{q=0}^{N_{H}} \xi_{q}^{\mu \nu}(k) e^{-i \omega_{q}(k) t} \\
& +\sum_{q=0}^{N_{N H}} \Xi_{q}^{\mu \nu}(k) e^{-i \Omega_{q}(k) t}+\text { b.c. }
\end{aligned}
$$

In writing the spectral decomposition (A5), we have deformed our original integration contour along the real $\omega$-axis to isolate the contributions coming from the singularities of $\hat{G}^{\mu \nu, \alpha \beta}(\omega, k)$ in the lower half of the complex $\omega$-plane. $N_{H}$ and $N_{N H}$ refer, respectively, to the number of hydrodynamic $\omega_{q}$ and nonhydrodynamic $\Omega_{q}$ modes excited by the metric fluctuation, while the excitation coefficients $\xi_{q}^{\mu \nu}$ and $\Xi_{q}^{\mu \nu}$ are determined by the residues of the retarded correlator at its poles and the initial data. Finally, b.c. denotes the continuous contributions coming from the branch cuts that might be present. These contributions are absent in theories of causal relativistic hydrodynamics and holography in the semiclassical limit but do appear in kinetic theory $[32,50]$.

As a final comment about (A5), note that we have also assumed that any remaining contribution coming from an integral around infinity can be neglected. This is justified in the case in which our microscopic theory is a conformal field theory and $t>0$; for $|\omega| \rightarrow \infty$, the retarded correlator should reduce to the vacuum result, which does not grow exponentially fast in the same limit.

Imagine now that $f^{\mu \nu}(x)$ is a square-integrable function supported only for $|x| \leq R$. Relativistic causality demands that, at $t>0$, the support of $\delta\left\langle T^{\mu \nu}(t, x)\right\rangle$ is at most $R+t$. Let us assume that $\delta\left\langle T^{\mu \nu}(t, x)\right\rangle$ is also square integrable at all times. Then, the Paley-Wiener theorem [40] tells us that the spatial Fourier transform of $\delta\left\langle T^{\mu \nu}(t, x)\right\rangle, \delta\left\langle T^{\mu \nu}(t, k)\right\rangle$, is an entire function of exponential type at most $R+t$, also square-integrable along the real $k$-axis. We remind the reader that an entire function $f(z)$ is a function analytic everywhere in the complex $z$-plane and that an entire function of exponential type $\sigma$ is an entire function obeying the bound

$$
|f(z)| \leq C e^{\sigma|z|}, \quad \forall z \in \mathbb{C}, \quad C \in \mathbb{R}^{+} .
$$


In the light of the Paley-Wiener theorem, and when the spectral decomposition (A5) holds, property 1 follows by contradiction: if the frequency $\omega(k)$ were entire, its Laurent series expansion

$$
\omega(k)=\sum_{n=1}^{\infty} w_{n} k^{n}
$$

would be convergent $\forall k \in \mathbb{C}$, and the bound (A6), as applied to $\delta\left\langle T^{\mu \nu}(t, k)\right\rangle$, would be violated. This result is in line with the conclusions of Ref. [51]. Since $\omega_{\perp}$ is given by a Taylor series in $k^{2}$, while $\omega_{\|}^{ \pm}$are series in $k$, the only possible exception to this behavior would be the case in which $\omega_{\perp}=0,\left|\omega_{\|}^{ \pm}\right| \propto|k|$, which corresponds precisely to ideal hydrodynamics.

On the other hand, property 2 can be justified as follows: if $\omega(k)$ had a pole, $\delta\left\langle T^{\mu \nu}(t, k)\right\rangle$ would develop an essential singularity at the pole location, thus failing to be entire. Furthermore, as argued in Ref. [51], for systems with a finite number of modes, a pole in some dispersion relation entails that the initial value problem does not have a unique solution.

A final consequence of property 2 is that nonhydrodynamic modes must exist in a theory that respects relativistic causality. These modes, which in principle could be absent if the singularities in the hydrodynamic dispersion relations were poles, appear naturally when analytically continuing these functions past the branch cuts that are actually present.

\section{APPENDIX B: THE TEMPORAL FORMULATION OF THE HYDRODYNAMIC GRADIENT EXPANSION}

Our objective in this Appendix is to provide a formulation of the gradient-expanded constitutive relations in terms of time derivatives. The elementary tensor structures this gradient expansion is built upon are $\sigma_{i j}$ and $\pi_{i j}^{\epsilon}$; since we are working in the Landau frame, there is no need to include $\pi_{i j}^{u}$, as the $t$-component of the conservation equation $\partial_{\mu} T^{\mu \nu}=$ 0 allows one to trade $\partial_{i} u^{i}$ by $\partial_{t} \epsilon$. Hence, we write

$$
\Pi_{i j}=-\tilde{A}\left(\partial_{t}\right) \sigma_{i j}-\tilde{B}\left(\partial_{t}\right) \pi_{i j}^{\epsilon}
$$

where

$$
\tilde{A}\left(\partial_{t}\right)=\sum_{n=0}^{\infty} \tilde{a}_{n} \partial_{t}^{n},
$$

and similarly for $\tilde{B}\left(\partial_{t}\right)$. Here and in the rest of this section, the tildes indicate quantities appearing in this temporal gradient expansion.

A matching computation analogous to the one presented in the main text allows one to express the transport coefficients $\tilde{a}_{n}$ and $\tilde{b}_{n}$ in terms of the hydrodynamic dispersion relations $k_{\perp}^{2}(\omega), k_{\|}^{2}(\omega)$ of the microscopic theory,

$$
\begin{gathered}
\tilde{a}_{n}=s T i^{n+1}\left[\omega^{n}\right]\left(\frac{\omega}{k_{\perp}^{2}(\omega)}\right), \\
\tilde{b}_{n}=i^{n}\left[\omega^{n}\right]\left(\frac{d-1}{d-2} \frac{\omega^{2}-\frac{k_{\|}^{2}(\omega)}{d-1}}{k_{\|}^{4}(\omega)}-\frac{2 \omega^{2}}{k_{\|}^{2}(\omega) k_{\perp}^{2}(\omega)}\right) .
\end{gathered}
$$

By (7), the low-order transport coefficients in this formulation are

$\tilde{a}_{0}=\eta, \quad \tilde{a}_{1}=\frac{s T \theta_{1}}{2 \eta}-\eta \tau_{\pi}, \quad \tilde{b}_{0}=\frac{\theta_{1}}{(d-1) \eta}$.

While the hydrodynamic dispersion relations are typically provided by expressing $\omega$ as a function of $k$, the formulation we are considering here has also appeared before in the literature (see Refs. [52,53] for a discussion in the holographic context).

We observe that, unlike the purely spatial formulation, the large-order behavior of the transport coefficients in the temporal gradient expansion is not governed by $\omega(k)$ but by the functions appearing inside the parentheses in (B3) and (B4). In cases where there are no branch points, these functions have poles corresponding to nonhydrodynamic modes at $k=0$, and hence the large-order behavior of the transport coefficients will be controlled by the nonhydrodynamic mode frequency with the smallest absolute value at $k=0$. This indicates that the natural condition for convergence in this case relies on the support of the solution in $\omega$ being below a certain $\tilde{\omega}_{*}$. One may be tempted to translate this natural formulation from a condition on support in $\omega$ to a condition on support in $k$ for the hydrodynamic fields. However, since this step relies on using the dispersion relations, the final condition in $k$ will likely be nonuniversal and potentially not be expressible as a single inequality. This points to the condition in $\omega$ being the most natural formulation for the temporal expansion, and we anticipate other natural conditions arising for other expansions. We leave this issue for future work.

The above analysis is illustrated in the following example for Müller-Israel-Stewart theory. The dispersion relation $k_{\perp}^{2}(\omega)$ is given there by

$$
k_{\perp}^{2}(\omega)=\frac{\omega\left(i+\tau_{\pi} \omega\right)}{D},
$$

and therefore $a_{n}=(-1)^{n} \tau_{\pi}^{n} \eta$. Moreover, the sound channel dispersion relation $k_{\|}^{2}(\omega)$ is such that $\tilde{b}_{n}=0$ and hence, in the temporal formulation, only $\sigma_{i j}$ appears in (B1). For the shear channel, Eq. (B6) gives rise to a pole in the function appearing in (B3) giving $\tilde{\omega}_{*}=1 / \tau_{\pi}$. In this simple 
example, this can be straightforwardly converted into a condition for support in $k,\left|k_{\max }\right|<\left|\tilde{k}_{*}\right|$, where

$$
\left|\tilde{k}_{*}\right|=\frac{1}{\sqrt{D \tau_{\pi}}}
$$

Note that this is proportional to, but distinct from, the critical momentum $k_{*}$ arising in the purely spatial expansion, given by (29). We can analogously define $\omega_{*}$, which is distinct from $\tilde{\omega}_{*}$. For $\left|k_{\max }\right|<\left|\tilde{k}_{*}\right|$, the gradient expansion is convergent, but showing this is subtle; unless the nonhydrodynamic mode present in the system is initially turned off, the $n$th term in the gradient expansion is not exponentially suppressed with the order but rather decays as a power law. This is due to the fact that the nonhydrodynamic mode evaluates to $-i \tilde{\omega}_{*}$ at $k=0$.
[1] P. Kovtun, Lectures on hydrodynamic fluctuations in relativistic theories, J. Phys. A 45, 473001 (2012).

[2] S. A. Hartnoll, A. Lucas, and S. Sachdev, Holographic Quantum Matter (MIT Press, Cambridge, Massachusetts, 2018) [arXiv:1612.07324].

[3] W. Florkowski, M. P. Heller, and M. Spaliński, New theories of relativistic hydrodynamics in the LHC era, Rep. Prog. Phys. 81, 046001 (2018).

[4] P. Romatschke and U. Romatschke, Relativistic Fluid Dynamics In and Out of Equilibrium, Cambridge Monographs on Mathematical Physics (Cambridge University Press, Cambridge, England, 2019).

[5] U. Heinz and R. Snellings, Collective flow and viscosity in relativistic heavy-ion collisions, Annu. Rev. Nucl. Part. Sci. 63, 123 (2013).

[6] W. Busza, K. Rajagopal, and W. van der Schee, Heavy ion collisions: The big picture, and the big questions, Annu. Rev. Nucl. Part. Sci. 68, 339 (2018).

[7] M. Shibata, K. Kiuchi, and Y.-i. Sekiguchi, General relativistic viscous hydrodynamics of differentially rotating neutron stars, Phys. Rev. D 95, 083005 (2017).

[8] M. G. Alford, L. Bovard, M. Hanauske, L. Rezzolla, and K. Schwenzer, Viscous Dissipation and Heat Conduction in Binary Neutron-Star Mergers, Phys. Rev. Lett. 120, 041101 (2018).

[9] F. S. Bemfica, M. M. Disconzi, and J. Noronha, Causality of the Einstein-Israel-Stewart Theory with Bulk Viscosity, Phys. Rev. Lett. 122, 221602 (2019).

[10] M. M. Caldarelli, O. J. Dias, R. Emparan, and D. Klemm, Black holes as lumps of fluid, J. High Energy Phys. 04 (2009) 024.

[11] S. R. Green, F. Carrasco, and L. Lehner, Holographic Path to the Turbulent Side of Gravity, Phys. Rev. X 4, 011001 (2014).

[12] R. Baier, P. Romatschke, D. T. Son, A. O. Starinets, and M. A. Stephanov, Relativistic viscous hydrodynamics, conformal invariance, and holography, J. High Energy Phys. 04 (2008) 100.

[13] P. K. Kovtun and A. O. Starinets, Quasinormal modes and holography, Phys. Rev. D 72, 086009 (2005).

[14] M. P. Heller, R. A. Janik, and P. Witaszczyk, Hydrodynamic Gradient Expansion in Gauge Theory Plasmas, Phys. Rev. Lett. 110, 211602 (2013).
[15] A. Buchel, M. P. Heller, and J. Noronha, Entropy production, hydrodynamics, and resurgence in the primordial quark-gluon plasma from holography, Phys. Rev. D 94, 106011 (2016).

[16] M. Baggioli and A. Buchel, Holographic viscoelastic hydrodynamics, J. High Energy Phys. 03 (2019) 146.

[17] A. Buchel, Non-conformal holographic Gauss-Bonnet hydrodynamics, J. High Energy Phys. 03 (2018) 037.

[18] I. Aniceto, B. Meiring, J. Jankowski, and M. Spaliński, The large proper-time expansion of Yang-Mills plasma as a resurgent transseries, J. High Energy Phys. 02 (2019) 073.

[19] M. P. Heller and M. Spaliński, Hydrodynamics Beyond the Gradient Expansion: Resurgence and Resummation, Phys. Rev. Lett. 115, 072501 (2015).

[20] G. Başar and G. V. Dunne, Hydrodynamics, resurgence, and transasymptotics, Phys. Rev. D 92, 125011 (2015).

[21] I. Aniceto and M. Spaliński, Resurgence in extended hydrodynamics, Phys. Rev. D 93, 085008 (2016).

[22] G. S. Denicol and J. Noronha, Divergence of the ChapmanEnskog expansion in relativistic kinetic theory, arXiv: 1608.07869.

[23] M. P. Heller, A. Kurkela, M. Spaliński, and V. Svensson, Hydrodynamization in kinetic theory: Transient modes and the gradient expansion, Phys. Rev. D 97, 091503 (2018).

[24] M. P. Heller and V. Svensson, How does relativistic kinetic theory remember about initial conditions? Phys. Rev. D 98, 054016 (2018).

[25] G. S. Denicol and J. Noronha, Exact Hydrodynamic Attractor of an Ultrarelativistic Gas of Hard Spheres, Phys. Rev. Lett. 124, 152301 (2020).

[26] L. P. Kadanoff and P. C. Martin, Hydrodynamic equations and correlation functions, Ann. Phys. (N.Y.) 24, 419 (1963).

[27] B. Withers, Short-lived modes from hydrodynamic dispersion relations, J. High Energy Phys. 06 (2018) 059 .

[28] S. Grozdanov, P. K. Kovtun, A. O. Starinets, and P. Tadić, Convergence of the Gradient Expansion in Hydrodynamics, Phys. Rev. Lett. 122, 251601 (2019).

[29] S. Grozdanov, P. K. Kovtun, A. O. Starinets, and P. Tadić, The complex life of hydrodynamic modes, J. High Energy Phys. 11 (2019) 097. 
[30] S. Grozdanov and N. Kaplis, Constructing higher-order hydrodynamics: The third order, Phys. Rev. D 93, 066012 (2016).

[31] S. M. Diles, L. A. Mamani, A. S. Miranda, and V.T. Zanchin, Third-order relativistic hydrodynamics: Dispersion relations and transport coefficients of a dual plasma, J. High Energy Phys. 05 (2020) 019.

[32] P. Romatschke, Retarded correlators in kinetic theory: Branch cuts, poles and hydrodynamic onset transitions, Eur. Phys. J. C 76, 352 (2016).

[33] S. Grozdanov, N. Kaplis, and A. O. Starinets, From strong to weak coupling in holographic models of thermalization, J. High Energy Phys. 07 (2016) 151.

[34] Y. Bu and M. Lublinsky, All order linearized hydrodynamics from fluid-gravity correspondence, Phys. Rev. D 90, 086003 (2014).

[35] Y. Bu and M. Lublinsky, Linearized fluid/gravity correspondence: From shear viscosity to all order hydrodynamics, J. High Energy Phys. 11 (2014) 064.

[36] M. Lublinsky and E. Shuryak, Improved hydrodynamics from the AdS/CFT duality, Phys. Rev. D 80, 065026 (2009).

[37] Y. Bu and M. Lublinsky, Linearly resummed hydrodynamics in a weakly curved spacetime, J. High Energy Phys. 04 (2015) 136.

[38] Y. Bu, M. Lublinsky, and A. Sharon, Hydrodynamics dual to Einstein-Gauss-Bonnet gravity: All-order gradient resummation, J. High Energy Phys. 06 (2015) 162.

[39] H.S. Wilf, generatingfunctionology (A K Peters, Ltd., Wellesley, Massachusetts, 2005).

[40] R.S. Strichartz, A Guide to Distribution Theory and Fourier Transforms (World Scientific, Singapore, 2003).
[41] B. Y. Levin, Lectures on Entire Functions, Translations of Mathematical Monographs, Vol. 150 (American Mathematical Soc., Providence, Rhode Island, 1996).

[42] M. P. Heller, Holography, Hydrodynamization and HeavyIon Collisions, in Proceedings, 56th Cracow School of Theoretical Physics: A Panorama of Holography: Zakopane, Poland, 2016, https://doi.org/10.5506/APhysPolB.47.2581; Acta Phys. Pol. B 47, 2581 (2016).

[43] I. Muller, Zum Paradoxon der Warmeleitungstheorie, Z. Phys. 198, 329 (1967).

[44] W. Israel, Nonstationary irreversible thermodynamics: A causal relativistic theory, Ann. Phys. (N.Y.) 100, 310 (1976).

[45] W. Israel and J. Stewart, Transient relativistic thermodynamics and kinetic theory, Ann. Phys. (N.Y.) 118, 341 (1979).

[46] M. P. Heller, A. Serantes, M. Spaliński, V. Svensson, and B. Withers, Transseries for causal diffusive systems, J. High Energy Phys. 04 (2021) 192.

[47] M. Beneke, Renormalons, Phys. Rep. 317, 1 (1999).

[48] M. P. Heller, A. Serantes, M. Spaliński, V. Svensson, and B. Withers (to be published).

[49] I. Aniceto, G. Basar, and R. Schiappa, A primer on resurgent transseries and their asymptotics, Phys. Rep. 809, 1 (2019).

[50] A. Kurkela and U. A. Wiedemann, Analytic structure of nonhydrodynamic modes in kinetic theory, Eur. Phys. J. C 79, 776 (2019).

[51] E. Krotscheck and W. Kundt, Causality criteria, Commun. Math. Phys. 60, 171 (1978).

[52] I. Amado, C. Hoyos-Badajoz, K. Landsteiner, and S. Montero, Absorption lengths in the holographic plasma, J. High Energy Phys. 09 (2007) 057.

[53] I. Amado, C. Hoyos-Badajoz, K. Landsteiner, and S. Montero, Hydrodynamics and beyond in the strongly coupled $N=4$ plasma, J. High Energy Phys. 07 (2008) 133. 